\title{
Confinement effects on strongly polar alkylcyanobiphenyl liquid crystals probed by dielectric spectroscopy
}

\author{
Jan Leys, Christ Glorieux and Jan Thoen \\ Laboratorium voor Akoestiek en Thermische Fysica, Departement Natuurkunde en \\ Sterrenkunde, Katholieke Universiteit Leuven, Celestijnenlaan 200D_-bus 2416, \\ B-3001 Leuven, Belgium \\ E-mail: jan.leys@fys.kuleuven.be and jan.thoen@fys.kuleuven.be
}

Received 27 February 2008

Published 29 May 2008

Online at stacks.iop.org/JPhysCM/20/244111

\begin{abstract}
Dielectric spectroscopy has often been used to study confinement effects in alkylcyanobiphenyl liquid crystals. In this paper, we highlight some of the effects that have been discovered previously and add new data and interpretation. Aerosil nanoparticles form a hydrogen bonded random porous network. In dispersions of alkylcyanobiphenyls with aerosils, an additional slow process arises, that we ascribe to the relaxation of liquid crystal molecules in close interaction with these nanoparticles. Their relaxation is retarded by a hydrogen bond interaction between the cyano group of the liquid crystals and an aerosil surface hydroxyl group. A similar surface process is also observed in Vycor porous glass, a random rigid structure with small pores. A comparison of the temperature dependence of the relaxation times of the surface processes in decylcyanobiphenyl and isopentylcyanobiphenyl is made, both for Vycor and aerosil confinement. In decylcyanobiphenyl, the temperature dependence for the bulk and surface processes is Arrhenius (in a limited temperature range above the melting point), except in Vycor, where it is a Vogel-Fulcher-Tamman dependence (over a much broader temperature range). In bulk and confined isopentylcyanobiphenyl, the molecular processes have a Vogel-Fulcher-Tamman dependence, whereas the surface processes have an Arrhenius one. Another effect is the acceleration of the rotation around the short molecular axis in confinement, and particularly in aerosil dispersions. This is a consequence of the disorder introduced in the liquid crystalline phase. The disorder drives the relaxation time towards a more isotropic value, resulting in an acceleration for the short axis rotation.
\end{abstract}

\section{Introduction}

Confined liquid crystals (LCs) have been an active research field in recent years. Apart from the fundamental interest in the behavior of a partially ordered fluid when it is restricted on the nano-level, glass physicists also show an interest in these experiments. In particular, theoreticians have argued that the presence of disorder in such systems leads to the formation of glasses, for example the so-called smectic glasses $[1,2]$. Apart from these more advanced considerations, a number of liquid crystals are themselves glass-forming liquids. We note the well-studied case of CB15 [3-8], the example of $5 \mathrm{CB}[9]$ and a recent comparison of the dielectric and thermal relaxation behavior in the E7 liquid crystal mixture [10]. These considerations drive the application of liquid crystals in the interesting area of glass physics.

Multiple types of confinement have been used and multiple techniques have been applied to confined LCs. In 1996, a book on the subject appeared, reviewing the knowledge available at that time [11]. We will select a number of results from the literature, without aiming to be complete. Important examples are calorimetric studies on aerogel systems [12, 13], where shifts in the phase transition temperatures and the appearance of double, broadened heat capacity peaks were the main observations. Comparable results were observed in aerosil dispersions [14-16], however, the behavior was somewhat different because of the more flexible nature of the aerosil network as compared to aerogels. A review 
on calorimetric data for $8 \mathrm{CB}$ is given in [17]. Structural information was obtained from x-ray diffraction $[16,18]$ and NMR [19-21]. These measurements gave information on the alignment of LCs in Anopore membranes [22], the disorder in Vycor porous glass [23], and disordering effects as well as certain critical phenomena at the phase transitions in aerosil dispersions. Disorder was also the main subject in a number of optical experiments and computer simulations [24-26]. The general picture, put forward by all these results, is that the close interaction with the surfaces of the confining materials results in a number of changes which can mainly be understood by the formation of domains and the disturbance of the long range order.

Also dielectric spectroscopy has been applied, elucidating aspects of the dynamics and the structure of confined LCs [7, 27-37]. This paper will attempt to make a synthesis of a number of dielectric results on alkylcyanobiphenyl liquid crystals in different types of confining systems, in particular Anopore membranes, Vycor porous glass and aerosil dispersions. A substantial part of these results have been published before, but they will be complemented with new data and interpretation.

\section{Experimental details}

The work presented mainly discusses our results from broadband dielectric spectroscopy. The data have been acquired by standard analyzers such as the Novocontrol Alpha Analyzer (for the low frequency range, $10^{-2}-10^{7} \mathrm{~Hz}$ ) and the Hewlett-Packard HP4291B Impedance Analyzer (for the high frequency range, $10^{6}-10^{9} \mathrm{~Hz}$ ). Most of the measurements were performed in cells specifically designed for dielectric measurements on liquid crystals, with modifications for the alignment of the sample. Details of the measurement procedures can be found elsewhere [7, 30, 35, 36, 38, 39].

We have used three types of confining structures, namely Anopore membranes, Vycor porous glass and aerosil porous networks. Anopore is a porous filter membrane, with regular cylindrical channels of $200 \mathrm{~nm}$ diameter. For the preparation of a sample, a piece of Anopore is first cleaned by heating it under vacuum. After cleaning, it is immersed in the isotropic LC, which fills the pores by the capillary force. The excess LC on the outside is removed before measuring. Vycor is a porous glass with a random network of interconnected channels, with a typical diameter of $7 \mathrm{~nm}$. Also in this case, after cleaning, isotropic LC is allowed to fill the pores by the capillary force. Aerosils are $7 \mathrm{~nm}$ diameter silicon oxide spheres, covered on the outside with hydroxyl groups. The groups can form hydrogen bonds with neighboring particles, resulting, at sufficient concentration, in a percolating network. Hydrophilic aerosils can be converted into hydrophobic aerosils. The preparation of a sample starts by drying the aerosils in a vacuum oven. The required amounts of LC and aerosil are mixed in a solvent and carefully dispersed. Then the solvent is allowed to evaporate slowly, while keeping the LC in the isotropic phase. In this way a homogeneous mixture of aerosil and LC is formed, resembling, depending on the concentration, a toothpaste-like to powder-like gel. The final step is to heat this product under vacuum again to remove the final traces of solvent [15].

\section{Some general notes on dielectric spectroscopy on cyanobiphenyl liquid crystals}

The liquid crystals discussed here will be mainly alkylcyanobiphenyls ( $n \mathrm{CBs}$ ), a class of LCs that have a dipole moment (located in the $-\mathrm{CN}$ terminal group) that is mainly oriented along the long molecular axis. This means that when the probing electric field lies in the same direction as the nematic director (parallel alignment), the relaxation spectrum is dominated by the process associated with the rotation of the molecule around the short molecular axis. In the same way, in perpendicular alignment, the probing electric field is perpendicular to the nematic director and mostly contributions related to the perpendicular component of the dipole moment will be probed. However, as this component is small in $n \mathrm{CBs}$, the perpendicular relaxation process is often interpreted as a librational motion of the molecule around the director, and not as the rotation around the long molecular axis (which is for example active in a molecule like MBBA [40] or D7AOB [41]). The fact that in each orientation one process dominates, makes it easier to interpret data. For example, the ratio of the strength of the short axis rotation and the libration can be seen as an estimate of the order in the sample.

This simplicity makes the cyanobiphenyls useful molecules, in addition they are stable substances that are quite easily available. However, when considering data concerning cyanobiphenyls, one should be aware of the presence of dipole correlations in these compounds. It has been shown that the cyanobiphenyls form dynamically antiparallel correlated pairs: the dipoles of the molecules align themselves antiparallel, partly canceling each other. This explains the existence of the Smectic- $A_{d}$ phase (with a layer spacing of the order of 1.4 times the molecular length) in 8CB [42] and the dielectric pretransitional effect observed in several cyanobiphenyls [43-47], where the static value of the dielectric permittivity bends away from the expected $1 / T$ behavior and even goes down as it approaches the isotropic-nematic transition. This effect reflects the enhanced dipole correlation in the pre-nematic fluctuations in the isotropic phase. It can be expected that confinement has an influence on the dipole correlations and thus the dielectric properties.

\section{The slow process in aerosil dispersions}

In 2001, Hourri et al [30] reported, in a dielectric study of 7CB with different concentrations of hydrophilic aerosils, that there is an extra relaxation process, slower than the main relaxation process in $7 \mathrm{CB}$, as depicted in figure 1. The main features of this new slow process that can be observed in this paper are: (i) its presence in both the nematic and isotropic phase, (ii) a retardation of about 2 decades compared to the rotation around the short molecular axis, and (iii) a dielectric strength that scales with aerosil concentration.

The process was explained as a surface process. Given observation (i), it does not take place in a part of the sample 


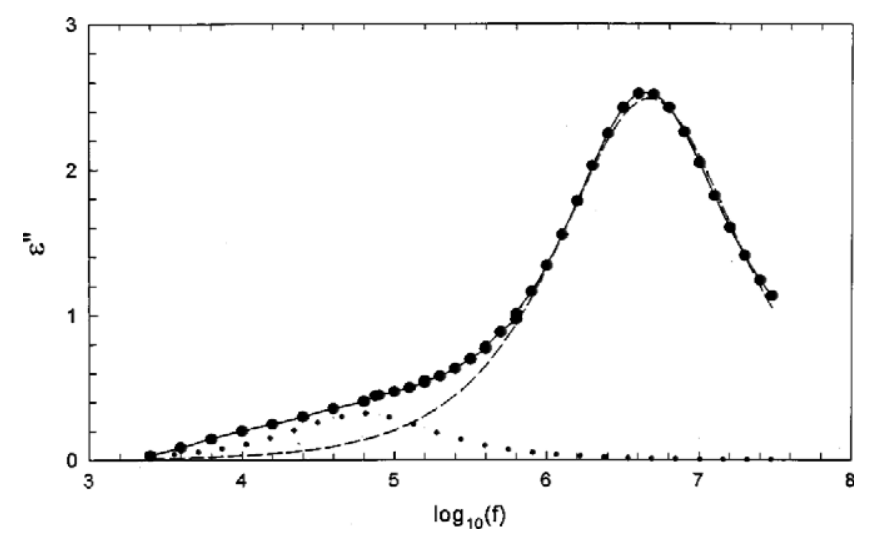

Figure 1. Spectrum of 7CB with $\rho_{s}=0.312 \mathrm{~g} \mathrm{~cm}^{-3}$ at $30.680^{\circ} \mathrm{C}$. See also [30].

sensitive to the phase transition; this is confirmed by the fact that the temperature dependence of the relaxation times did not alter when crossing the NI transition. Also observation (iii) fits in this view: the higher the aerosil concentration, the more important the surface layer is and the stronger a surface process becomes. Reference [30] also shows that the temperature dependence is comparable to that of the bulk relaxation, suggesting a comparable relaxation mechanism. At that time, the authors supposed (but did not mention in the paper) that a hydrogen bond, between the terminal $-\mathrm{CN}$ group of the liquid crystal molecules and the surface $-\mathrm{OH}$ group of the aerosil particles, was responsible for the slow process. The molecules first have to overcome this hydrogen bond before they can reorient themselves in the electric field, resulting in a slower relaxation process. Later Frunza et al showed by FTIR spectroscopy that such a hydrogen bond actually exists $[32,48]$.

The slow process was further investigated in a number of liquid crystals, mainly in $n \mathrm{CBs}$, although it was also observed in a weakly polar LC, namely D6AOB [38]. Generally the picture introduced by Hourri et al was confirmed. For example, the slow process was also found in 5CB [35] and in $10 \mathrm{CB}[36,39]$, with the same features as observed previously in 7CB. In [35], aerosil dispersions were confined to Anopore membranes, and also in these doubly confined systems, the slow process is visible; this observation is an extra argument for its nature as a surface process. The slow process is also found in $\mathrm{CB} 15$, the chiral isomer of 5CB [7]. We will explore this case further in section 6 , and compare it to the situation in $10 \mathrm{CB}$.

The previous studies mainly used hydrophilic aerosils, however, a number of experiments on dispersions with hydrophobic aerosils have also been performed [7, 38]. The main observation here is that the slow process is also found in these cases, however, considerably higher concentrations of hydrophobic aerosils are needed before the same dielectric strength is reached as for hydrophilic aerosils. We can compare for example the dielectric strengths for the dispersions reported in [7] shown in figure 2, where the value of the dielectric strength of the surface process is normalized to the strength of the rotation around the short axis and plotted versus the

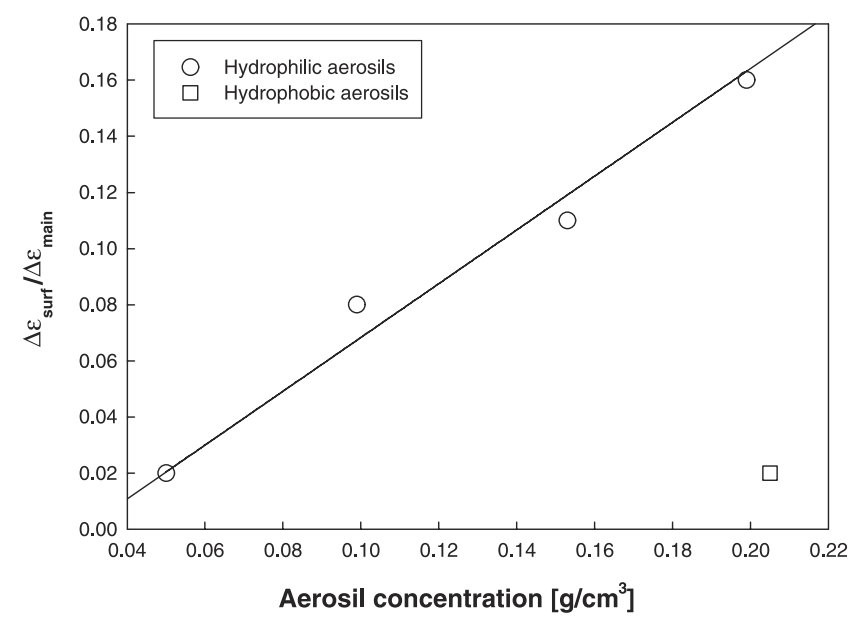

Figure 2. Comparison of the dielectric strengths of the surface process normalized to the strength of the main relaxation process, as observed in CB15. The line is a linear fit to the hydrophilic data. Data according to [7].

aerosil concentration. The ratio of the dielectric strengths roughly measures the fraction of molecules participating in the surface process ${ }^{1}$. The dielectric strength increases linearly with concentration for the hydrophilic aerosils. For the hydrophobic aerosils, the dielectric strength is about $1 / 4$ of that for the same concentration of the hydrophilic aerosils. This ratio corresponds to the relative number of surface $-\mathrm{OH}$ groups present in both types of aerosils. In the production process for hydrophobic aerosils, only $3 / 4$ of the $-\mathrm{OH}$ groups on the hydrophilic aerosils are eliminated [49]. The remaining $-\mathrm{OH}$ groups are still available for hydrogen bonding with the LC molecules. This observation is also qualitatively visible in [38], where the strength of the process in the hydrophilic aerosils is such that one cannot expect a four times weaker process in the hydrophobic aerosils to be visible; this is exactly what is observed. This correspondence between the dielectric strength and the surface properties is again a convincing argument for the interpretation as a surface process.

\section{CB15 confined to Vycor porous glass}

We studied the effects of confinement to Vycor on the chiral glass-forming monotropic liquid crystal CB15. This work complements earlier work on CB15 with aerosils [7], and allows for the comparison with 10CB that we will make in section 6. A typical spectrum is given in figure 3. At low frequencies, the contribution of conductivity dominates. Then a process is visible, not present in bulk, that, based on the analogy with the surface process in aerosils (section 4) and the surface process in 10CB in Vycor [36], should be considered as a surface process. At higher frequencies the relaxation due to the molecular rotation is visible. We were unable to split this process into the rotation around the long and the short molecular axis in the supposed chiral nematic phase temperature region, as was possible for CB15 with

1 It ignores the contribution of the long axis process, which increases with increasing concentration (because of the increasing disorder). 


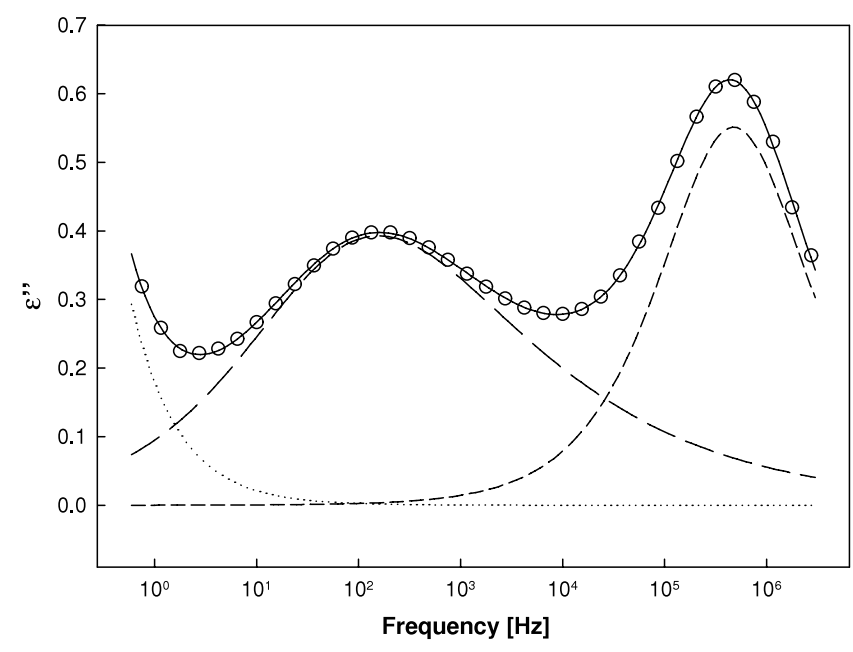

Figure 3. Spectrum of CB 15 confined to Vycor porous glass at 267.15 K. The solid line is a fit with two Havriliak-Negami terms and a conductivity term. Short dash: molecular relaxation process; long dash: surface process; dotted: conductivity.

aerosils and for 10CB in Vycor. This indicates that the liquid crystalline order in CB15 in Vycor is severely disturbed, resulting in a isotropic-like dynamical response over the entire temperature range measured. Both processes mentioned are clearly not Debye processes, substantial symmetric and asymmetric broadening was observed for the surface process; for the molecular process, the symmetric broadening was much larger than the asymmetric broadening.

The results for the relaxation times can be found in figure 4. For the molecular process, the phase transition to a supercooled chiral nematic phase is absent. The relaxation is also clearly accelerated compared to the bulk rotation around the short axis. This is consistent with the results for 10CB in Vycor, and this acceleration can probably be understood by the same arguments as the acceleration in aerosils, to be discussed in section 7. The temperature dependence shows the curvature typical for a VFT dependence. The relaxation times of the surface process are somewhat slower than for the aerosil surface process. We have no clear understanding of the reason for this change, as on comparison with $10 \mathrm{CB}$, this is not the case. More important is that the temperature dependence is different from that of the molecular process, it appears closer to an Arrhenius behavior than a VFT, especially when realizing that the data are most reliable between 3.6 and $4.0 \times 10^{-3} \mathrm{~K}^{-1}$ (see figure 4).

\section{Surface processes compared: $\mathrm{CB} 15$ versus $10 \mathrm{CB}$, aerosil versus Vycor}

When we studied the relaxation processes for $10 \mathrm{CB}$ and for $10 C B$ in different types of confinement [36], we noted that within the available temperature range the relaxation processes in bulk 10CB and in Anopore and aerosil confinement were Arrhenius processes. However, careful analysis of the relaxation processes in Vycor showed that only a VFT temperature dependence could give a satisfactory fit to the relaxation times: the two molecular processes and the surface

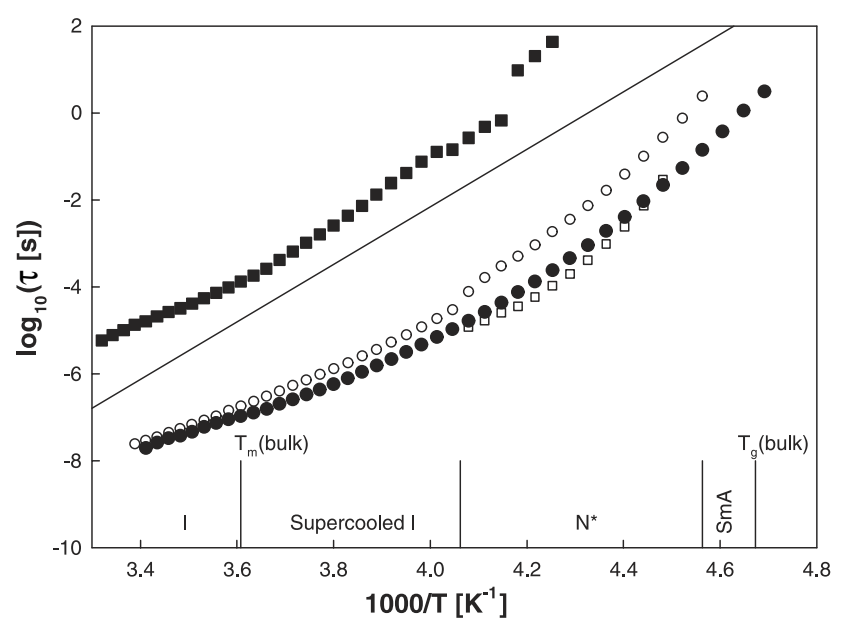

Figure 4. Relaxation times for the molecular relaxation processes in bulk CB15: O: short axis rotation, $\square$ : long axis rotation; molecular process in CB15 in Vycor; $\square$ : surface process in Vycor; the straight line is an average for the surface process in aerosils, based on the values of [7].

process were VFT processes. This was an unexpected observation, as in the case of CB15, we observed that, although CB15 has a clear VFT dependence in bulk, the aerosil surface process could be best interpreted as an Arrhenius process [7]. In order to elucidate this situation, we investigated the surface process in CB15 confined to Vycor porous glass. This gives us the possibility of comparing the surface processes in both CB15 and 10CB, and also both in aerosils and in Vycor.

Figure 4 shows the relaxation times for a number of processes in CB15 and confined CB15. The data for the relaxations in the bulk and for the aerosils are taken from [7]. We have replaced the individual data points for the slow relaxation process in the different aerosil samples by a straight line. The relaxation times for CB15 in Vycor are discussed in section 5 .

In figure 5, we present the relaxation time data for the processes in 10CB and in confined 10CB, close to the isotropic to smectic A (IA) phase transition (for bulk $10 \mathrm{CB}$ at $50.2^{\circ} \mathrm{C}$ ). The bulk relaxation times show a phase transition at $3.10 \times$ $10^{-3} \mathrm{~K}^{-1}$. For the relaxation times of the rotation around the short axis in Vycor, the transition is nearly invisible, and is situated at about $3.17 \times 10^{-3} \mathrm{~K}^{-1}$. It is clear that this rotation is accelerated compared to the same rotation in bulk $10 \mathrm{CB}$ in the liquid crystalline phase. In the isotropic phase the difference is negligible. The surface processes are all about two decades slower than the bulk relaxation processes. The slow aerosil processes have the same temperature dependence and nearly the same characteristic frequency, and are insensitive to the phase transition, as expected (see section 4). The surface process in Vycor has a different temperature dependence, it evolves more like the bulk processes than the aerosil surface processes. It also does not experience the phase transition.

A third result that should be included in this discussion is that of Frunza et al [32], who applied dielectric spectroscopy to dispersions of $n \mathrm{CBs}(n=5-8)$ with very high aerosil concentrations, up to regimes where it can be expected that 


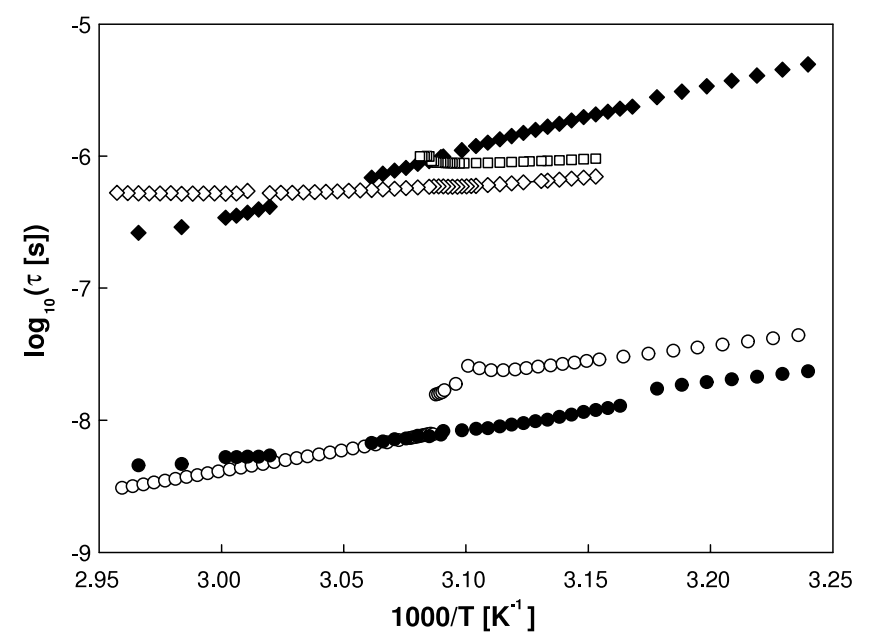

Figure 5. Relaxation times for the short axis rotation process in bulk $10 \mathrm{CB}$ and 10CB in Vycor, and for the surface process in aerosils and in Vycor. O: bulk 10CB, short axis rotation; $\diamond: 10 \mathrm{CB}$ with $8 \%$ aerosils, surface relaxation; $\square$ : $10 \mathrm{CB}$ with $31 \%$ aerosils, surface relaxation; $10 \mathrm{CB}$ in Vycor, short axis rotation; $\$$ : $10 \mathrm{CB}$ in Vycor, surface relaxation. Data after [36].

almost every LC molecule is interacting with the aerosil surface. They conclude that in such a case, the surface process has a VFT dependence, for measurements over a broad temperature range (about -50 to $120^{\circ} \mathrm{C}$ ).

Thus, in conclusion we have the following observations: for the 'ordinary' $n \mathrm{CBs}$, if the surface process can be tracked over a sufficient temperature range, it shows a VFT dependence, but, when viewed over a much more limited temperature range, it appears to have an Arrhenius dependence. This explains the apparent difference between the reported temperature dependence for the surface process in 10CB with aerosils as compared to the $n \mathrm{CBs}(n=5-8)$ with very high aerosil concentrations. If we had been able to supercool $10 \mathrm{CB}$ we would also have seen a VFT dependence for the surface process. Such a VFT dependence is observed for the surface process of 10CB confined to Vycor, confirming the view that the VFT dependence is the 'correct' temperature dependence for these LCs.

In the same respect, the confinement of 10CB in Vycor also prevented the crystallization of the bulk part of the sample, allowing us to investigate the (of course influenced by the confinement) values of the bulk relaxation times. Also these values show a VFT dependence, confirming the statements given above. Comparable observations can also be found in other studies, for example for 5CB in Anopore membranes [27], where supercooling was achieved. The relaxation plot shows that the relaxation frequencies have a bending that could be interpreted as an VFT dependence.

While this reasoning explains the temperature dependencies that have been observed in the literature, one question remains. Since we can assume that $n \mathrm{CBs}$ and $\mathrm{CB} 15$ have a VFT dependence for their molecular relaxation processes, it is difficult to understand why the surface relaxation processes have: in the case of the $n$ CBs a VFT dependence, whereas in CB15 the surface processes in both aerosil and Vycor confinement are practically Arrhenius processes.
In the report on CB15-aerosil dispersions [7], an explanation is put forward in terms of the Adam-Gibbs relation and cooperatively reorienting regions (CRRs) [50]. The Adam-Gibbs relation can be stated as

$$
\tau=\tau_{0} \exp \left(\frac{n \Delta \mu}{k T}\right),
$$

where $\tau$ is the relaxation time, $\tau_{0}$ the limiting high temperature relaxation time, $\Delta \mu$ the potential barrier hindering the cooperative rearrangement per molecule and $n$ the number of molecules in a CRR. Although this relationship is somewhat contentious (see e.g. [51]), the basic picture it provides is plausible. The Adam-Gibbs relation provides some theoretical support for the VFT equation: in a glass-forming material, the size of the CRR increases with decreasing temperature, which results in a VFT-like curve. If the size of the CRR and the potential barrier do not change with temperature, the Adam-Gibbs relation reduces to the Arrhenius temperature dependence. It makes sense to consider the size of the CRR for the surface process as being constant with temperature, because we do not see how the surface layer can change its size with temperature. Also, the potential barrier probably stays the same. This means that for a surface process, an Arrhenius temperature dependence can be expected on the grounds of the Adam-Gibbs relation and the reasonable assumptions that the size of the surface layer and the potential barrier for reorientation do not change.

In case of the other $n \mathrm{CBs}$, the surface processes in both Vycor and aerosils show a VFT dependence, indicating that one of the above assumptions concerning the CRR size or the potential barrier is not correct. However, it is not clear to us what the difference could be between CB 15 and the other $n \mathrm{CBs}$ that explains this behavior.

\section{Acceleration of the short axis rotation in aerosils}

In many dielectric studies on confined liquid crystals, changes in the relaxation times of the molecular rotation around the short axis have been reported. This change is typically an acceleration, and has been reported in diverse systems, most notably Anopore membranes [27], Synpore membranes [52], Vycor glass (this work), and aerosil dispersions. In fact, this acceleration was already mentioned in what appears to be the first dielectric study on aerosil dispersions [53]. However, later studies have not always reported it, although it was often visible in the figures. Two years ago, we published an extensive overview of the properties of the rotation around the short axis of 5CB in different types of confinement [35]. This allowed us to propose a model to explain this acceleration in the liquid crystalline phase. Around the same time, another group reported the existence of the acceleration in the nematic phase of a weakly polar crystal [37]. This indicates that an explanation of the phenomenon should use quite general concepts, independent of the polarity of the LC. We will now verify whether our hypothesis of isotropization, presented in [35], can be applied in the situations found in the literature.

Isotropization is a consequence of the disorder that is introduced by the aerosils in a liquid crystalline phase. 
The aerosil network divides the liquid crystal molecules over domains, which of course disrupts the long range liquid crystalline order. This means that the structure, and consequently also the dynamics, will be driven towards a more isotropic one. One of the effects of the liquid crystalline order is a retardation of the rotation around the short axis compared to the isotropic phase. If the order is disrupted, this rotation should become faster again, evolving in the direction of the isotropic value with increasing disorder, or equivalently, increasing aerosil concentration. We realize that we are not presenting a quantitative model for the acceleration, however, it does explain qualitatively the features observed in 5CB [35] and also those for a weakly polar LC [37].

In fact, the model as presented above also makes a prediction concerning the fast process, and the libration or the rotation around the long molecular axis. This faster process is faster in the liquid crystalline phases compared to the isotropic phase, meaning that it should slow down under the influence of aerosil-induced disorder. This prediction was verified in 5CB [54]. The same paper also shows that the accelerating effect is also visible in dispersions of 5CB with hydrophobic aerosils (all previously measured results concerned hydrophilic aerosils).

If we look back in the literature, we see that the acceleration is reported or at least visible from the figures in many of the publications [35-37, 39, 53-57], so it appears to be a rather common effect. Isotropization gives a basic understanding of the phenomenon. However, in [35], it is clear that the acceleration is also present in the isotropic phase. Since the isotropic phase cannot be disordered, it is clear that isotropization cannot be applied in this case. We think that a possible explanation can be found in the distortion of the dipole correlation between two molecules, as described earlier in section 3. Due to the dipole correlation, a molecule has not only to overcome the normal rotational viscosity, related to the presence of the neighboring molecules, but also the force emerging from this electrostatic interaction. This means that the relaxation of a molecule in such an antiparallel correlated pair should be somewhat slower than the relaxation of a corresponding monomer. When the formation of such correlated pairs is distorted, as in the case of aerosils under the influence of the hydrogen bonding capability of aerosil particles, the rotation should slightly accelerate. Such a behavior roughly corresponds to what is observed in the case of $5 \mathrm{CB}$ in [35]. However, further investigation of the acceleration in the isotropic phase is necessary to verify this hypothesis.

\section{Conclusion}

Dielectric spectroscopy data on confined cyanobiphenyls show a number of changes in comparison to the bulk systems, indicating surface and disordering effects on these partially ordered fluids.

(i) The emergence of surface relaxation processes. Confining systems offer large surface areas to the materials they host, and the interaction of liquid crystals with this surface leads to the appearance of slow surface relaxation processes in Vycor porous glass and in dispersions with aerosil nanoparticles. In this last case, the hydrogen bond between the cyano end group and the surface hydroxyl groups leads to a retarded relaxation in a surface layer around the particles. In Vycor a comparable mechanism is probably active.

(ii) A change in the dynamical behavior of the liquid crystal molecules in Vycor. This is observed for CB15 confined to Vycor, where the bulk relaxation modes clearly have a VFT dependence of their relaxation times on temperature, whereas the surface processes both in aerosil dispersions and Vycor show an activated Arrhenius dependence. This is opposite to the behavior observed in the other alkylcyanobiphenyls.

(iii) An acceleration of the rotation around the short molecular axis. Although this can be observed in multiple types of confinement, the results of aerosil dispersions show that acceleration is a consequence of the disorder that is introduced by the confining matrix, resulting in a disruption of the liquid crystalline order and an evolution of the dynamics to one more resembling that of the isotropic phase.

\section{Acknowledgments}

This work was financially supported by the Fund for Scientific Research Flanders (Belgium) (FWO Contract G.0125.03N) and by K. U. Leuven (Contract GOA-02). JL acknowledges the Research Fund of K U Leuven for a postdoctoral fellowship (PDM/07/098).

\section{References}

[1] Jacobsen B, Saunders K, Radzihovsky L and Toner J 1999 Phys. Rev. Lett. 831363

[2] Radzihovsky L and Toner J 1999 Phys. Rev. B 60206

[3] Massalska-Arodz M, Williams G, Smith I K, Conolly C, Aldridge G A and Dabrowski R 1998 J. Chem. Soc., Faraday Trans. 94387

[4] Drozd-Rzoska A, Rzoska S J, Zioło J and Czupryński K 1999 J. Phys.: Condens. Matter 11 L473

[5] Mayer J, Massalska-Arodz M and Krawczyk J 2001 Mol. Cryst. Liq. Cryst. 366211

[6] Rzoska S J, Paluch M, Pawlus S, Drozd-Rzoska A, Ziolo J, Jadzyn J, Czuprynski K and Dabrowski R 2003 Phys. Rev. E 68031705

[7] Sinha G, Glorieux C and Thoen J 2004 Phys. Rev. E 69031707

[8] Drozd-Rzoska A, Rzoska S J, Paluch M, Pawlus S, Ziolo J, Santangelo P G, Roland C M, Czuprynski K and Dabrowski R 2005 Phys. Rev. E 71011508

[9] Zeller H R 1982 Phys. Rev. Lett. 48334

[10] Bràs A R, Dionísio M, Huth $\mathrm{H}$, Schick $\mathrm{Ch}$ and Schönhals A 2007 Phys. Rev. E 75061708

[11] Crawford G P and Zumer S (ed) 1996 Liquid Crystals in Complex Geometries Formed by Polymer and Porous Networks (London: Taylor and Francis)

[12] Wu L, Zhou B, Garland C W, Bellini T and Schaefer D W 1995 Phys. Rev. E 512157

[13] Kutnjak Z and Garland C W 1997 Phys. Rev. E 55488

[14] Zhou B, Iannacchione G S, Garland C W and Bellini T 1997 Phys. Rev. E 552962

[15] Haga H and Garland C W 1997 Phys. Rev. E 563044 
[16] Iannacchione G S, Garland C W, Mang J T and Rieker T P 1998 Phys. Rev. E 585966

[17] Iannacchione G S 2004 Fluid Phase Equilib. 222/223 177

[18] Leheny R L, Park S, Birgeneau R J, Gallani J-L, Garland C W and Iannacchione G S 2003 Phys. Rev. E 67011708

[19] Zeng H, Zalar B, Iannacchione G S and Finotello D 1999 Phys. Rev. Е 605607

[20] Jin T and Finotello D 2001 Phys. Rev. Lett. 86818

[21] Jin T and Finotello D 2004 Phys. Rev. E 69041704

[22] Crawford G P, Steele L M, Ondris-Crawford R, Iannacchione G S, Yeager C J, Doane J W and Finotello D 1992 J. Chem. Phys. 967788

[23] Iannacchione G S, Crawford G P, Qian S, Doane J W, Finotello D and Zumer S 1996 Phys. Rev. E 532402

[24] Bellini T, Buscaglia M, Chiccoli C, Mantegazza F, Pasini P and Zannoni C 2000 Phys. Rev. Lett. 851008

[25] Bellini T, Buscaglia M, Chiccoli C, Mantegazza F, Pasini P and Zannoni C 2002 Phys. Rev. Lett. 88245506

[26] Rotunno M, Buscaglia M, Chiccoli C, Mantegazza F, Pasini P, Bellini T and Zannoni C 2005 Phys. Rev. Lett. 94097802

[27] Rozanski S A, Stannarius R, Groothues H and Kremer F 1996 Liq. Cryst. 2059

[28] Cramer C, Cramer T, Kremer F and Stannarius R 1997 J. Chem. Phys. 1063730

[29] Sinha G P and Aliev F M 1998 Phys. Rev. E 582001

[30] Hourri A, Bose T K and Thoen J 2001 Phys. Rev. E 63051702

[31] Aliev F M 2002 J. Non-Cryst. Solids 307-310 489

[32] Frunza S, Frunza L, Tintaru M, Enache I, Beica T and Schönhals A 2004 Liq. Cryst. 31913

[33] Bengoechea M R and Aliev F M 2005 J. Non-Cryst. Solids 3512685

[34] Aliev F M, Bengoechea M R, Gao C Y, Cochran H D and Dai S 2005 J. Non-Cryst. Solids 3512690

[35] Sinha G, Leys J, Glorieux C and Thoen J 2005 Phys. Rev. E 72051710

[36] Leys J, Sinha G, Glorieux C and Thoen J 2005 Phys. Rev. E 71051709

[37] Lobo C V, Krishna Prasad S and Yelamaggad C V 2006 J. Phys.: Condens. Matter 18767
[38] Sinha G, Oka A, Glorieux C and Thoen J 2004 Liq. Cryst. 311123

[39] Sinha G, Leys J, Glorieux C and Thoen J 2005 J. Non-Cryst. Solids 3512780

[40] Buka A, Owen P G and Price A H 1979 Mol. Cryst. Liq. Cryst. 51295

[41] Oka A, Sinha G, Glorieux C and Thoen J 2004 Liq. Cryst. 3131

[42] Leadbetter A J, Frost J C, Gaughan J P, Gray G W and Mosley A 1979 J. Phys. 40375

[43] Bradshaw M J and Raynes E P 1981 Mol. Cryst. Liq. Cryst. Lett. 7273

[44] Thoen J and Menu G 1983 Mol. Cryst. Liq. Cryst. 97163

[45] Thoen J and Bose T K 1999 Handbook of Low and High Dielectric Constant Materials and Their Applications ed H S Nalwa (San Diego, CA: Academic)

[46] Van Roie B, Leys J, Denolf K, Glorieux C, Pitsi G and Thoen J 2005 Phys. Rev. E 72041702

[47] Leys J, Glorieux C, Wübbenhorst M and Thoen J 2007 Liq. Cryst. $\mathbf{3 4} 749$

[48] Frunza L, Kosslick H, Bentrup U, Pitsch I, Fricke R, Frunza S and Schönhals A 2003 J. Mol. Struct. 651-653 341

[49] Degussa Corp. 2003 Technical Bulletin Fine Particles No.11: Basic Characteristics of AEROSIL ${ }^{\circledR}$ Fumed Silica

[50] Adam G and Gibbs J H 1965 J. Chem. Phys. 43139

[51] Ngai K L 2000 J. Non-Cryst. Solids 2757

[52] Różański S A, Stannarius R and Kremer F 1999 Z. Phys. Chem. 211147

[53] Tschierske S, Yaroshchuk O V and Kresse H 1995 Cryst. Res. Technol. 30571

[54] Rozanski S A, Sinha G P and Thoen J 2006 Liq. Cryst. 33833

[55] Aliev F M, Sinha G P and Kreuzer M 2001 Mol. Cryst. Liq. Cryst. 359537

[56] Abd-El-Messieh S L, Werner J, Schmalfuss H, Weissflog W and Kresse H 1999 Liq. Cryst. 26535

[57] Hourri A, Jamée P, Bose T K and Thoen J 2002 Liq. Cryst. 29459 